\title{
1 Ageing in house sparrows is insensitive to 2 environmental effects
}

${ }^{1}$ Department of Animal and Plant Sciences, University of Sheffield, Sheffield, S10

$92 \mathrm{TN}, \mathrm{UK}$

$10{ }^{2}$ Department of Life Sciences, Imperial College London, London, SW7 2AZ, UK

$11{ }^{3}$ Evolutionary Biology, Max Planck Institute for Ornithology, Seewiesen, DE-

12 82319, Germany

$13{ }^{4}$ Evolution \& Ecology Research Centre and School of Biological, Earth and

14 Environmental Sciences, University of New South Wales, Sydney, NSW 2052,

15 Australia

\section{*shared last author}

Running title: Ageing in wild and captive sparrows

Keywords: Senescence, mark-recapture, demography, wild, Passer domesticus, evolution, ecology, island, mortality, aging

Word count main text: 3,718

Number of references: 57

Number of figures: 4

\section{Number of tables: 3}

Correspondence to: Mirre Simons, Department of Animal and Plant Sciences, University of Sheffield, Sheffield, S10 2TN, UK

34 Email: m.simons@sheffield.ac.uk 


\section{Abstract}

36 Variation in individual life histories, and physiology, determines the rates at

37 which new life is generated (reproduction) and lost (death) in a population.

38 Studying the demography of deaths thus reveals fundamental aspects of the

39 biology of individuals within a population. We studied mortality senescence - the

40 increase in mortality rate with age - in wild and captive house sparrows (Passer

41 domesticus), and demonstrate highly similar mortality senescence in both, but

42 markedly lower vulnerability to death (frailty) in captivity. This suggests that

43 house sparrows have a species-specific rate of ageing that is insensitive to

44 environmental effects. Unexpectedly, juvenile and adult mortality co-varied

45 positively across years in the wild, indicating that mortality is not strongly

46 density-dependent. Mortality also varied widely among years, suggesting a

47 strong environmental effect, and we explain the observed patterns using

48 temperature data and predation by birds of prey. We discuss how stochastic

49 environmental effects can affect the evolution of ageing.

50 


\section{Introduction}

52 Demography can reveal unique aspects of the biology of the individuals within a

53 population ${ }^{1-5}$. An interest in the demography of wild populations has recently

54 been ignited ${ }^{6-8}$, driven by the question of whether wild animals show

55 senescence. Historically, senescence was presumed to be minimal in the wild,

56 swamped by extrinsic mortality (e.g. predation, disease, accidents, harsh

57 weather) such that very few individuals in the wild would live long enough to

58 show senescence ${ }^{8,9}$. This hypothesis has now been falsified by studies in a

59 multitude of wild populations in which evidence has been found for both

60 reproductive and mortality senescence ${ }^{8,10,11}$. In many of these studies the

61 confounding effect of an unknown rate of dispersal with age is an important

62 limitation, with dispersal being mistaken for mortality. The number of studies of

63 age-specific survival remains, however, severely limited, especially compared to

64 the many on reproductive senescence. Studying both factors together can

65 provide a unique contribution to understanding the evolution of senescence in a

66 comparative context ${ }^{7,8}$. Recent comparisons of mortality trajectories across

67 species have revealed a wide range of patterns, from negligible senescence to a

68 rapid acceleration in mortality with age, and this variation currently remains

69 largely unexplained ${ }^{12}$.

70

71 Variation in mortality trajectories among species can result from differences in

72 physiology and also from environmental effects and their interactions with

73 physiology ${ }^{13}$. The demography of death records reveals two main components:

74 the increasing risk of death with age - the ageing rate - and frailty, the 
75 vulnerability to death from ageing-related causes ${ }^{14-16}$. These parameters have

76 both genetic and environmental components. Studies on insects, utilizing the

77 advantage of obtaining many related offspring within a lineage, have reported

78 heritability for both these parameters ${ }^{1,17}$. Another approach is to estimate the

79 scope for environmental effects by comparing different populations of the same

80 species or comparing mortality in the wild with that in captivity. Baboons (Papio

81 hamadryas) in two different wild populations and a captive population have

82 been shown to have different levels of frailty, yet to show a highly similar rate of

83 ageing ${ }^{18}$. Similarly, the recent gain in human lifespan ${ }^{19}$ is due to a reduction in

84 frailty, rather than a change in ageing rate ${ }^{20}$. Together, these studies suggest that

85 there are both environmental and genetic determinants of frailty. Environmental

86 effects on ageing rate are less common, although diet has been shown to

87 modulate ageing rate in rodents ${ }^{16}$.

88

89 The underlying physiology of differential adult mortality trajectories, and

90 especially the magnitude of the latter compared to juvenile mortality, results

91 from selection pressures shaped by life-history trade-offs. Adult and offspring

92 mortality risks are expected to be traded-off with the costs of reproduction,

93 determining, for instance, the optimal clutch size and effort that should be

94 invested in provisioning 21 . Interestingly, separating juvenile and adult survival is

95 not usually possible because of dispersal in non-closed populations ${ }^{22,23}$. This

96 limits our ability to understand the connection between demography and life-

97 history trade-offs, and also to detect and quantify the density dependence of

98 population dynamics ${ }^{22}$. For example, it would be difficult in a non-closed 
population to separate an effect of density on survival from an effect on density-

100 dependent dispersal.

102 Here, we study the demography of an exceptionally well-monitored, closed

103 island population 24,25 , and also of a captive population, of house sparrows ${ }^{26}$

104 (Passer domesticus) to (i) compare mortality senescence in the wild and in

105 captivity. We also (ii) assess the effect of the environment on adult mortality and

106 juvenile recruitment in the wild, factors that we were able to separate reliably

107 due to the near-perfect resighting rate, comprehensive coverage of breeding

108 attempts on the island, and negligible rates of immigration and emigration ${ }^{24,27}$.

\section{Methods}

\section{Study populations}

112 Monitoring of the house sparrows on Lundy Island $\left(51.10^{\circ} \mathrm{N}, 4.40 \mathrm{~W}^{\circ}\right)$, England,

113 began in 1990 and has been undertaken systematically since 2000; data are

114 presented here for the period 2000-2012. Every year, breeding behaviour in and

115 outside nestboxes is monitored and birds are trapped using mistnets during each

116 winter (Nov-Feb). Captures include those by researchers focusing on sparrows

117 specifically, and those caught by the Lundy Field Society during bird surveys.

118 Individuals are ringed before fledging with individual metal rings and a unique

119 combination of colour rings, allowing sightings without actual captures, and

120 individuals are sampled for blood as chicks and adults for genetic parentage

121 analyses ${ }^{10,28}$. To estimate the population size and resighting probability in our

122 study population we used actual catches ${ }^{25}$, ad libitum sightings during the 
123 breeding season and winter, structured sightings from social parentage

124 assignment of broods using video recording 27 and genetic sightings (genetic

125 parentage assignment to offspring, except for the last year included, 2012, for

126 which pedigree information was not yet available at the time of analysis). We

127 assessed juvenile survival for all nestlings that received a metal British Trust for

128 Ornithology ring, which they were given at an age of $c a 12$ days to focus on post-

129 fledging survival, and we disregarded earlier deaths in the nest. Lundy is a small

130 island ( $<5.0 \mathrm{~km}$ long and $0.7 \mathrm{~km}$ wide) and is $19 \mathrm{~km}$ from the closest mainland

131 shoreline, which limits dispersal from and to the island to almost non-existent ${ }^{24}$.

132 The habitat on Lundy consists of a small village and farm surrounded mostly by

133 grassland and cliffs, but with a small wooded valley. The sparrows are almost

134 exclusively restricted to the village and the adjacent wooded valley; an excess of

135 nestboxes was available throughout the study area. Predators consisted of

136 occasional birds of prey that pass through during migration or, occasionally,

137 become resident on the island in winter (see below).

139 The captive population of sparrows was maintained at the Max Planck Institute

140 for Ornithology (Seewiesen, Germany) from 2005 (results include data on

141 mortality up to 2014). Individuals were originally captured from the wild in rural

142 Bavaria, Germany and subsequently maintained in aviaries. A proportion of the

143 offspring born in captivity were maintained in the population and inbreeding

144 was avoided as much as possible by transferring birds among aviaries. All birds

145 were individually ringed and mortality was monitored daily. The specific

146 husbandry of the birds and previous research has been described elsewhere ${ }^{26,29}$. 
147 The captive dataset consisted of 304 adult birds, including 170 individuals that

148 were still alive and were therefore right-censored.

\section{Mortality trajectory and resighting probability}

151 We used Bayesian Survival Trajectory Analysis (BaSTA, 1.9.2) implemented in

sampling for survival parameters and latent states (when times of birth or death are unknown) in a mark-recapture framework. Mark-recapture models use the missed observations of individuals known to be alive at the point of missed observation (i.e. they are observed later) to estimate the probability that an individual is sighted in the population. BaSTA combines such mark-recapture probability modelling with fitting the mortality/survival trajectory.

160 We fitted Gompertz and Logistic models with a bathtub shape ${ }^{30,32}$. Gompertz and

161 Logistic models differ, in that Logistic models allow the mortality rate to plateau 162 at advanced ages, whereas under the Gompertz law mortality rate continuously 163 accelerates exponentially with age ${ }^{30,32}$. An increase in mortality rate with age is 164 evidence for senescence. A bathtub shape (declining Gompertz, see equation 1) 165 allowed early mortality, from the nestling state to adulthood, to be modelled. We 166 selected the best model based on the deviance information criterion (DIC, which

167 behaves similarly to AIC), and checked convergence by running each chain eight 168 times, with each individual chain run for 1,000,000 iterations, with a burnin of 1692,000 and thinning interval of 2,000. Autocorrelations of the chain were below

1700.045 for all parameters in all models run. We included sex as a categorical

171 covariate (allowing mortality parameters to vary between the sexes) in the 
models, because sex differences in longevity are prevalent across the animal

173 kingdom ${ }^{33}$. The inclusion of this covariate might therefore improve the fit of the

174 BaSTA model and thus the estimation of the re-sighting probability and

parametric survival models. The significance of the inclusion of sex as a covariate was judged using the Kullback-Leibner divergence calibration ${ }^{11,30}$, which ranges

177 from 0.5 to 1 , with 0.5 indicating identical posterior distributions, and hence no

178 effect of the covariate. We included known birth years (i.e. observed as a chick),

179 where possible, for 2,297 of the 2,514 individuals included, for which we had

181 from recoveries made in the field (for 155 individuals). Sighting years were

182 coded from 1 March until 29 February in the following year in order to include

183 sightings up to the start of the next breeding season. Mortality trajectories in the

184 captive population were fitted using the package 'flexsurv' in R in a maximum-

185 likelihood framework ${ }^{34}$. Individuals that died as a result of accidents in the population, and those still alive, were right-censored. We only fitted adult mortality, because data for juvenile (under one year old) mortality were not complete, because this was not always recorded in the required detail. The

189 parametric models fitted were limited to a Gompertz without any covariates, 190 because in the smaller captive dataset the Logistic model did not converge and 191 mortality deceleration was not evident in the raw data. For a direct comparison 192 with the wild population, a simple Gompertz without the bathtub structure was 193 also fitted in 'flexsurv' to the Lundy data. 


$$
h(t)=e^{a_{0}-a_{1} * t}+c+e^{b_{0}+b_{1} * t}
$$
model $^{30}$. The first part of the equation $\left(a_{0}, a_{1}, c\right)$ models the decline

$$
h(t)=e^{a_{0}-a_{1} * t}+c+\frac{e^{b_{0}+b_{1} * t}}{1+b_{2} * \frac{e^{b_{0}}}{b_{1}} *\left(e^{b_{1} * t}-1\right)}
$$
model ${ }^{30}$. The logistic model converges to the Gompertz model when parameter $b_{2}$, which allows mortality to decelerate with age, approaches zero.

\section{Predictors of mortality in the Lundy population}

\section{Temperature data}

215 We explored the relationships between the mortality of juveniles and resident

216 adults with temperature. Minimum daily temperature data were obtained from

217 the Meteorological Office (UK) for the weather station at RAF Chivenor $\left(51.1^{\circ} \mathrm{N}\right.$,

$2184.1 \mathrm{~W}^{\circ}$, the closest official weather station to Lundy, $35 \mathrm{~km}$ to the east). We

219 expected low temperatures to induce mortality. As a measure of how cold a year

220 was, we used the number of days per census year (from April to March, as above) 
221 that had a minimum temperature below the lower daily minimum temperature

222 quartile across the years 1999 to February 2012 (i.e. $\leq 4.6^{\circ} \mathrm{C}$ ).

\section{Presence of birds of prey}

225 To assess a possible relationship between juvenile or adult mortality and the

226 number of predators on the island, data on sightings of specified birds of prey

227 were used to construct a predator index for each census year. Sightings of these

228 species are routinely recorded by permanent island staff, members of the Lundy

229 Field Society and visitors to the island, and are collated into monthly sighting

230 numbers. We derived an annual index of all the sightings in each census year of

231 sparrowhawk (Accipter nisus), merlin (Falco columbarius), hobby (Falco

232 subbuteo) and kestrel (Falco tinnunculus) as the sum of the months in which

233 there were at least two sightings of a species, divided by 48 ( 4 species times 12

234 months). This predator index therefore represents the relative proportion of a

235 census year for which the population was at risk of predation. These raptor

236 species commonly visit Lundy during migration, but in some years remain as

237 residents, and their presence was therefore chosen as a proxy of the predation

238 pressure acting on the population. All four species are known predators of house

239 sparrows. In the case of the kestrel, which is generally known to have a

240 preference for vole species, it is known that the species switches its diet towards

241 passerines almost exclusively when the availability of small mammalian prey is

242 reduced ${ }^{35}$. The only small mammal currently present on Lundy is the pygmy

243 shrew (Sorex minutus), following the successful eradication of rats on the island

244 in 2002-2004. 


\section{Statistical model}

247 In order to separate the potentially shared covariance among the independent

248 variables tested against mortality in the population, and to also correct for

249 changes in age demography affecting adult mortality in a census year, linear

250 mixed effects binomial models were fitted in 'Ime4' in R. Two models were fitted,

251 one for juvenile and another for adult mortality, that included a random

252 intercept term for census year of the study and fixed terms for the three

253 independent variables considered. For adult mortality, the effect of age on

254 mortality was fitted as a factor, given the non-linear nature of this relationship

255 (Figure 1), and individuals aged over 5 years were pooled into a single category

256 to aid model convergence. 
257

258

\section{9}

260

261

262

263

264

265

266

267

268

269

270

271 Estimated recapture probabilities were close to saturation and highly similar

272

273

274

275

276

\section{7}

278

279

280

281

\section{Results}

\section{Mortality trajectories and resighting probability}

The Logistic model fitted the Lundy data best (Table 1, $\Delta$ DIC 32.9). The

magnitude of the b2 parameter, with its 95\% CI well above zero, indicated that mortality levelled off at the oldest ages, probably contributing to the superior fit of the Logistic model over the Gompertz model. There was no difference in the mortality trajectory between the sexes; Kullback-Leibner divergence calibrations remained very close to 0.5 for all parameters (range: $0.50-0.61$ ).

Mortality dropped steeply for adults that recruited into the population and there was a moderate acceleration in mortality with age, as indicated by the significantly positive values of the $b 1$, the ageing rate parameter, in both the Logistic and Gompertz models (Table 1).

across all models (deviation of 0.001 ), with very narrow confidence intervals (for the preferred Logistic model $=0.96,95 \%$ CI 0.95-0.97). Mortality in the captive population was lower and this was due to a change in the frailty parameter, with a highly similar rate of ageing in captivity and in the wild (Table 2, Figure 2).

\section{Detailed demography of juvenile and adult mortality}

The high re-sighting probability and the near absence of dispersal to and from the island ${ }^{24}$ allowed us to separate juvenile mortality from adult mortality.

Adults were assumed dead if not seen in the next year (ignoring the 4\%, based on the estimated re-sighting probability, that we are expected to miss in each 
282

283

284

285

286

287

288

289

290

291

292

293

294

295

296

297

census year) and thus recruited juveniles could be separated from adult survival.

Using this approach, we constructed a detailed picture of juvenile mortality and

adult mortality independently, across the years of our study. Year to year

variation in mortality (Figure 3) was high and statistically significant (logistic

regressions, juvenile: $\chi^{2}(11)=49, p<0.0001$, adult: $\left.\chi^{2}(11)=105, p<0.0001\right)$. Note

that some individual adults are used repeatedly in these analyses because they

were alive in multiple years, creating pseudoreplication; yet, given the relatively

short mean lifespan (Figure 1), we expect these effects to be relatively minimal.

Juvenile and adult mortality covaried positively across years $\left(r_{s}=0.70, \mathrm{p}=\right.$

0.015), indicating that when adult mortality was high, this was also the case for juveniles (Figure 3).

To explore any environmental effects on mortality, we investigated the effects of number of cold days per census year, population size, and predator index on adult mortality and juvenile mortality (Figure 4). Surprisingly, in cold years, adult and juvenile mortality was lower $\left(r_{s}=-0.65, \mathrm{p}=0.02 ; r_{s}=-0.52, \mathrm{p}=0.08\right.$, respectively). Adult mortality and juvenile mortality were each positively related to population size (adult + juveniles), but neither relationship reached significance (adult: $r_{s}=0.27, \mathrm{p}=0.39$; juvenile: $r_{s}=0.41, \mathrm{p}=0.19$ ). Predator index predicted juvenile $\left(r_{s}=0.61, \mathrm{p}=0.03\right)$ and adult mortality $\left(r_{s}=0.59, \mathrm{p}=\right.$ 0.04). In the binomial mixed effect models used to pull apart the independent effects of these three independent variables, predator presence exhibited the strongest effects, with only this variable reaching significance and then only in adults (Table 3). This is probably due to the covariance between predator presence and cold weather $\left(r_{s}=-0.38, \mathrm{p}=0.23\right)$, because separately each of these 
parameters did predict mortality significantly or showed a trend for both

308 juvenile and adult mortality (Table 3).

309

\section{Discussion}

\section{Actuarial senescence}

312 We detect relatively small but significant mortality senescence ('actuarial

313 senescence') in wild house sparrows (Figure 1). There was a $\sim 1.6$-fold increase

314 in mortality from its trough at age $\sim 2$ to age $\sim 6$; in comparison, there is a $\sim 3$ -

315 fold increase in mortality rates in a US human population from the age of 60 to

316100 years and a $\sim 2.7$-fold increase in mortality rate in male mice from the age of

3171.4 to 2.9 years $^{36}$. This level of actuarial senescence in the sparrow population is

318 detected despite strong environmental effects on adult and juvenile mortality.

319 This is relevant to the evolution of senescence, given that the strength (and

320 sometimes direction) of selection on life history is changed by the level of

321 stochastic (environmental) noise ${ }^{37,38}$. Extrinsic mortality shapes optimal

322 investment in the soma over reproduction (current versus future reproduction

323 trade-off) ${ }^{39}$ and, accordingly, different levels of extrinsic mortality on the

324 population level lead to different levels of senescence-related mortality ${ }^{14}$. When

325 this selective pressure - extrinsic mortality - is more variable, selection on

326 intrinsic, senescence-related, mortality is weaker and hence a larger standing

327 variation in intrinsic mortality is expected ${ }^{40}$. Moreover, different levels of

328 stochasticity can also lead to the evolution of differential bet-hedging

329 strategies ${ }^{41}$. 
330 Studies of the fitness costs of senescence ${ }^{7}$ and evolutionary theory of ageing ${ }^{42}$

331 have not considered environmental stochasticity. Different levels of

332 environmental stochasticity might also explain differences between species in

333 the demography of fertility and mortality ${ }^{12,43}$. Understanding the effects of

334 environmental variation in extrinsic mortality on intrinsic mortality and/or their

335 interactions will be a next step in understanding the evolution of senescence in

336 the wild. In addition, such considerations might explain why the rates of

337 reproductive senescence and mortality senescence do not always match $7,14,44,45$,

338 perhaps because the selective pressures maintaining them are differentially

339 susceptible to environmental effects. Effects of the developmental environment

340 on reproductive, but not survival senescence ${ }^{13}$ is perhaps an illustration of such

341 differential environmental effects on life-history traits.

\section{Environmental effects on the demography of mortality}

344 House sparrows in captivity and in the wild show a highly similar rate of ageing

345 but differential frailty, consistent with similar comparisons in mammals, namely

346 baboons ${ }^{18}$ and humans ${ }^{20}$. This suggests that the ageing rate is a specific property

347 of a species, insensitive to environmental effects. An invariable within-species

348 rate of ageing is fundamental to the compensation law of mortality and the

349 mortality deceleration that follows from the reliability theory of ageing $16,46,47$.

350 Although comparative evidence from birds (this study) and mammals ${ }^{18,20}$,

351 including the mortality deceleration observed in this study and others ${ }^{4}$, now

352 point in this direction, in contrast, experimental evidence suggests that ageing

353 rate can be flexible. Dietary restriction in rodents reduces the rate of ageing

354 without affecting frailty ${ }^{16}$, contrary to, for example, the effect of dietary 
377 Indeed, population size in a census year did not impinge strongly on either juvenile or adult mortality (Table 3, Figure 4). Of the other two environmental

restriction on mortality in Drosophila melanogaster, which is exclusively attributable to a change in frailty ${ }^{48,49}$. Parental effort also modulates the ageing rate in the wild, as demonstrated using brood size manipulations in jackdaws (Corvus monedula $)^{11}$, the white-throated sparrow (Zonotrichia albicollis) ${ }^{50}$, and the Seychelles Warbler (Acrocephalus sechellensis) ${ }^{51}$. This suggests that, to some extent, the wide variation in mortality trajectories between species ${ }^{12}$ can be due to differential environmental or population effects ${ }^{52}$. Distinguishing between captive and wild populations ${ }^{53,54}$, and examining experimental effects on frailty and ageing rate independently, will be crucial steps towards understanding the evolution of mortality trajectories and senescence.

\section{Juvenile and adult mortality}

The high recapture probability we estimated from the BaSTA models allowed for the separation of juvenile and adult mortality, which in many other study systems is not possible. Juvenile and adult mortality were also found to be sensitive to environmental effects, and covaried positively, suggesting that juveniles and adults died of similar environmental causes. The analysis also suggests that any density dependent effect is relatively limited, given that adult survival does not impinge on juvenile mortality. Therefore, food availability and aggression, resulting from competition for food or mating territories, are unlikely to limit the population size.

$$
\text { variables, predator presence was most predictive of mortality, especially in }
$$


380 adults. When considered separately, cold weather was associated with improved

381 survival of juveniles and adults. Irrespective of whether this is indirect via

382 predation pressure, it goes against the usual expectation of harsher winters,

383 although still relatively mild on Lundy, causing more mortality. Without being

384 able to attribute causes of death to starvation, disease or directly to predation,

385 we can only speculate on the roles of physiology and/or ecology in the

386 relationship between mortality and ambient temperature.

387

388 Intensive monitoring, as in the Lundy house sparrow population, achieving near

389 perfect re-sighting rates, is key when inferring biology from the demography of

390 mortality, which is crucial in explaining the evolution ${ }^{12}$ and biology $3,55,56$ of

391 ageing. The confounding factors of dispersal and unmarked individuals that are

392 typical of most study populations would bias such estimates, and can lead to

393 potentially false conclusions about differences in demography between species

394 when the degree of these biases differs across study populations and species ${ }^{12}$.

395 Understanding the physiology and evolution of relatively invariable species-

396 specific ageing rates in the face of strong environmental effects, as we show here

397 for a bird in addition to existing evidence from mammals, is pivotal to

398 understanding natural selection on senescence and its physiology. 


\section{Acknowledgements}

402 We thank Ian Cleasby, Duncan Gillespie, Simon Griffith, Yu-Hsun Hsu, Maria

403 Karlsson, Maria-Elena Mannarelli, Nancy Ockendon and Claire Prosser for their

404 contributions to the fieldwork. We are grateful to Bart Kempenaers for providing

405 access to the captive population of house sparrows and for support. We thank

406 Emmi Schlicht and Silke Laucht from the Kempenaers Group for providing

407 mortality data for the population, and the animal caretakers for looking after the

408 birds. We thank the UK Meteorological Office for supplying the daily temperature

409 data and the Lundy Company staff for their enthusiastic support of our research.

410 The Lundy Field society and visitors to the island are thanked for recording and

411 collating sightings of birds on Lundy. Support by the UK Natural Environment

412 Research Council (NE/J024597/1 and NE/M005941/1 to TB). SN was supported

413 by a Rutherford Discovery Fellowship (New Zealand) and a Future Fellowship

414 (Australia). MJPS is supported by a Sir Henry Wellcome Fellowship and a

415 Sheffield Vice Chancellor's fellowship. TB was supported by a Leverhulme

416 Fellowship. 
417 1. Tatar, M. \& Carey, J. R. Genetics of Mortality in the Bean Beetle

418 Callosobruchus maculatus. Evolution (N. Y). 48, 1371 (1994).

419 2. Mair, W., Goymer, P., Pletcher, S. D. \& Partridge, L. Demography of dietary 420 restriction and death in Drosophila. Science 301, 1731-3 (2003).

421 3. Garratt, M., Nakagawa, S. \& Simons, M. J. P. Comparative idiosyncrasies in 422 life extension by reduced mTOR signalling and its distinctiveness from dietary restriction. Aging Cell 15, (2016).

424 4. Barbi, E., Lagona, F., Marsili, M., Vaupel, J. W. \& Wachter, K. W. The plateau 425 of human mortality: Demography of longevity pioneers. Science $\mathbf{3 6 0}$, 1459-1461 (2018).

5. Metcalf, C. J. E. \& Pavard, S. Why evolutionary biologists should be demographers. Trends Ecol. Evol. 22, 205-212 (2007).

6. Bronikowski, A. M. et al. Aging in the natural world: Comparative data reveal similar mortality patterns across primates. Science 331, 1325-1328 (2011).

7. Bouwhuis, S., Choquet, R., Sheldon, B. C. \& Verhulst, S. The Forms and Fitness Cost of Senescence: Age-Specific Recapture, Survival, Reproduction, and Reproductive Value in a Wild Bird Population. Am. Nat. 179, E15-E27 (2012).

8. Nussey, D. H., Froy, H., Lemaitre, J. F., Gaillard, J. M. \& Austad, S. N. its implications for bio-gerontology. Ageing Res. Rev. 12, 214-225 (2013). evolutionary ecology of senescence. Funct. Ecol. 22, 371-378 (2008).

10. Schroeder, J., Burke, T., Mannarelli, M. E., Dawson, D. A. \& Nakagawa, S. 
Maternal effects and heritability of annual productivity. J. Evol. Biol. 25, 149-156 (2012).

444 11. Boonekamp, J. J., Salomons, M., Bouwhuis, S., Dijkstra, C. \& Verhulst, S. Reproductive effort accelerates actuarial senescence in wild birds: an experimental study. Ecol. Lett. 17, 599-605 (2014).

12. Jones, O. R. et al. Diversity of ageing across the tree of life. Nature 505, 169-173 (2014).

13. Cooper, E. B. \& Kruuk, L. E. B. Ageing with a silver-spoon: A meta-analysis of the effect of developmental environment on senescence. Evol. Lett. 2, 460-471 (2018).

14. Ricklefs, R. E. Insights from comparative analyses of aging in birds and mammals. Aging Cell 9, 273-284 (2010).

15. Kirkwood, T. B. L. Deciphering death: a commentary on Gompertz (1825) 'On the nature of the function expressive of the law of human mortality, and on a new mode of determining the value of life contingencies'. Philos. Trans. R. Soc. B Biol. Sci. 370, 20140379-20140379 (2015).

16. Simons, M. J. P., Koch, W. \& Verhulst, S. Dietary restriction of rodents decreases aging rate without affecting initial mortality rate - a metaanalysis. Aging Cell 12, 410-414 (2013).

461 17. Promislow, D. E., Tatar, M., Khazaeli, A. A. \& Curtsinger, J. W. Age-specific patterns of genetic variance in Drosophila melanogaster. I. Mortality. Genetics 143, 839-48 (1996).

464 18. Bronikowski, A. M. et al. The aging baboon: Comparative demography in a non-human primate. Proc. Natl. Acad. Sci. 99, 9591-9595 (2002).

466 19. Oeppen, J. \& Vaupel, J. W. Demography: Broken limits to life expectancy. 
Science 296, 1029-1031 (2002).

468 20. Vaupel, J. W. Biodemography of human ageing. Nature 464, 536-542 (2010).

470 21. Stearns, S. C. Trade-Offs in Life-History Evolution. Funct. Ecol. 3, 259-268 (1989).

22. Sæther, B.-E. et al. Generation time and temporal scaling of bird population dynamics. Nature 436, 99-102 (2005).

23. Matthysen, E. Density-dependent dispersal in birds and mammals. Ecography 28, 403-416 (2005).

24. Schroeder, J., Nakagawa, S., Rees, M., Mannarelli, M.-E. \& Burke, T. Reduced fitness in progeny from old parents in a natural population. Proc. Natl. Acad. Sci. 112, 4021-4025 (2015).

25. Simons, M. J. P., Winney, I., Nakagawa, S., Burke, T. \& Schroeder, J. Limited catching bias in a wild population of birds with near-complete census information. Ecol. Evol. 5, 3500-3506 (2015).

26. Girndt, A., Chng, C. W. T., Burke, T. \& Schroeder, J. Male age is associated with extra-pair paternity, but not with extra-pair mating behaviour. Sci. Rep. 8, 8378 (2018). Provisioning. Am. Nat. 188, 219-230 (2016).

28. Winney, I. S. et al. Heritability and social brood effects on personality in juvenile and adult life-history stages in a wild passerine. J. Evol. Biol. 31, 75-87 (2018).

490 29. Laucht, S., Kempenaers, B. \& Dale, J. Bill color, not badge size, indicates testosterone-related information in house sparrows. Behav. Ecol. Sociobiol. 
64, 1461-1471 (2010).

493 30. Colchero, F., Jones, O. R. \& Rebke, M. BaSTA: An R package for Bayesian estimation of age-specific survival from incomplete markrecapture/recovery data with covariates. Methods Ecol. Evol. 3, 466-470 (2012).

31. Colchero, F. \& Clark, J. S. Bayesian inference on age-specific survival for censored and truncated data. J. Anim. Ecol. 81, 139-149 (2012).

32. Pletcher, S. D., Khazaeli, A. A. \& Curtsinger, J. W. Why Do Life Spans Differ? Partitioning Mean Longevity Differences in Terms of Age-Specific Mortality Parameters. Journals Gerontol. Ser. A Biol. Sci. Med. Sci. 55, B381-B389 (2000).

33. Clutton-Brock, T. . \& Isvaran, K. Sex differences in ageing in natural populations of vertebrates. Proc. R. Soc. B Biol. Sci. 274, 3097-3104 (2007).

506 34. Jackson, C. flexsurv : A Platform for Parametric Survival Modeling in R. J. Stat. Softw. 70, 1-33 (2016).

35. Yalden, D. W. Notes on the diet of urban kestrels. Bird Study 27, 235-238 (1980).

510 36. Gavrilova, N. S. \& Gavrilov, L. A. Biodemography of old-age mortality in humans and rodents. Journals Gerontol. - Ser. A Biol. Sci. Med. Sci. 70, 1-9 (2015).

513 37. Tuljapurkar, S. An uncertain life: Demography in random environments. Theor. Popul. Biol. 35, 227-294 (1989).

515 38. Benton, T. G. \& Grant, A. How to Keep Fit in the Real World: Elasticity Analyses and Selection Pressures on Life Histories in a Variable 
Environment. Am. Nat. 147, 115-139 (1996).

518 39. Kirkwood, T. B. L. \& Rose, M. R. Evolution of Senescence: Late Survival (1991).

521 40. Waxman, D. \& Peck, J. R. Sex and adaptation in a changing environment. Genetics 153, 1041-1053 (1999).

41. Childs, D. Z., Metcalf, C. J. E. \& Rees, M. Evolutionary bet-hedging in the real world: empirical evidence and challenges revealed by plants. Proc. R. Soc. B Biol. Sci. 277, 3055-3064 (2010).

42. Kowald, A. \& Kirkwood, T. B. L. Evolutionary significance of ageing in the wild. Exp. Gerontol. 71, 89-94 (2015).

43. Rees, M., Childs, D. Z., Rose, K. E. \& Grubb, P. J. Evolution of size-dependent flowering in a variable environment: construction and analysis of a stochastic integral projection model. Proceedings. Biol. Sci. 271, 425-34 (2004).

44. Ricklefs, R. E., Scheuerlein, A. \& Cohen, A. Age-related patterns of fertility in captive populations of birds and mammals. Exp. Gerontol. 38, 741-745 (2003).

45. Burger, J. M. S. \& Promislow, D. E. L. Are functional and demographic senescence genetically independent? Exp. Gerontol. 41, 1108-1116 (2006).

46. Gavrilov, L. A. \& Gavrilova, N. S. The reliability theory of aging and longevity. J. Theor. Biol. 213, 527-545 (2001).

539 47. Boonekamp, J. J., Simons, M. J. P., Hemerik, L. \& Verhulst, S. Telomere length behaves as biomarker of somatic redundancy rather than biological age. Aging Cell 12, 330-332 (2013). 
542

543

544

545

546

547

548

549

550

551

552

553

554

555

556

557

558

559

560

48. Mair, W., Goymer, P., Pletcher, S. D. \& Partridge, L. Demography of dietary restriction and death in Drosophila. Science 301, 1731-3 (2003).

49. McCracken, A. W., Adams, G., Hartshorne, L. \& Simons, M. J. P. The hidden costs of dietary restriction: implications for its evolutionary and mechanistic origins. bioRxiv Preprint, doi:10.1101/533711 (2019).

50. Grunst, M. L. et al. Actuarial senescence in a dimorphic bird: different rates of ageing in morphs with discrete reproductive strategies. Proc. R. Soc. B Biol. Sci. 285, 20182053 (2018).

51. Hammers, M. et al. Breeders that receive help age more slowly in a cooperatively breeding bird. doi:10.1038/s41467-019-09229-3

52. Roach, D. A. \& Gampe, J. Age-Specific Demography in Plantago : Uncovering Age-Dependent Mortality in a Natural Population. Am. Nat. 164, 60-69 (2004).

53. Ricklefs, R. E. Evolutionary Theories of Aging: Confirmation of a Fundamental Prediction, with Implications for the Genetic Basis and Evolution of Life Span. Am. Nat. 152, 24-44 (1998).

54. Turbill, C. \& Ruf, T. Senescence is more important in the natural lives of long- than short-lived mammals. PLoS One 5, (2010).

55. Garratt, M., Nakagawa, S. \& Simons, M. J. P. Life-span Extension With Reduced Somatotrophic Signaling: Moderation of Aging Effect by Signal Type, Sex, and Experimental Cohort. J. Gerontol. A. Biol. Sci. Med. Sci. 72, 1620-1626 (2017).

56. Partridge, L., Pletcher, S. D. \& Mair, W. Dietary restriction, mortality trajectories, risk and damage. Mech. Ageing Dev. 126, 35-41 (2005).

57. Blaker, H. Confidence curves and improved exact confidence intervals for 
bioRxiv preprint doi: https://doi.org/10.1101/598284; this version posted April 4, 2019. The copyright holder for this preprint (which was not

certified by peer review) is the author/funder, who has granted bioRxiv a license to display the preprint in perpetuity. It is made available under aCC-BY-NC-ND 4.0 International license.

567

discrete distributions. Can. J. Stat. 28, 783-798 (2000).

568

569 
Tables

Table 1. Comparison of parametric survival models of the wild house sparrows

573 on Lundy. The first three variables parameterise the bathtub shape $\left(a_{0}, a_{1}\right.$ and $c$;

574 Figure 1). The b set of adult mortality senescence parameters are defined

575 accordingly (see text and Equation 1 and 2).

\begin{tabular}{|l|l|l|}
\hline Parameter & Gompertz (95\% CI) & Logistic (95\% CI) \\
\hline $\mathbf{a}_{\mathbf{0}}$ & $0.378(0.150: 0.640)$ & $0.407(0.199: 0.633)$ \\
\hline $\mathbf{a}_{1}$ & $1.923(1.220: 2.876)$ & $1.599(0.944: 2.530)$ \\
\hline $\mathbf{c}$ & $0.356(0.095: 0.526)$ & $0.285(0.042: 0.499)$ \\
\hline $\mathbf{b}_{\mathbf{0}}$ ('frailty') & $-2.690(-4.368:-1.322)$ & $-3.224(-4.778:-1.828)$ \\
\hline $\mathbf{b}_{\mathbf{1}}$ ('ageing rate') & $0.327(0.154: 0.548)$ & $0.749(0.338: 1.282)$ \\
\hline $\mathbf{b}_{\mathbf{2}}$ ('deceleration') & & $1.045(0.144: 2.212)$ \\
\hline DIC & 6537 & 6504 \\
\hline
\end{tabular}

578 Table 2. Gompertz fits of adult mortality in the captive and wild population. Note

579 the high similarity in the ageing rate parameter $\left(b_{1}\right)$ and the substantial

580 difference in the frailty parameter $\left(b_{0}\right)$. Refer to Figure 2 for a plot of the models.

\begin{tabular}{|l|l|l|}
\hline $\begin{array}{l}\text { Gompertz } \\
\text { Parameter }\end{array}$ & Captive (95\% CI) & Wild (95\% CI) \\
\hline $\mathbf{b}_{\mathbf{0}}$ ('frailty') & $-3.117(-3.461:-2.769)$ & $-1.309(-1.423:-1.194)$ \\
\hline $\mathbf{b}_{\mathbf{1}}$ ('ageing rate') & $0.203(0.127: 0.270)$ & $0.228(0.188: 0.268)$ \\
\hline
\end{tabular}


582 Table 3. Estimates from the mixed binomial models, including census year as a

583 random effect, either testing the three environmental variables together in a full

584 model or separately, run for juvenile and adult mortality separately. Raw

585 estimates of scaled variables are given with their standard errors. ${ }^{* *}$ indicates $p<$

$5860.01, *$ indicates $p<0.05, \dagger$ indicates $p<0.1$. Models of adult mortality included

587 age to correct for differences in age demography and its associated mortality

588 between years.

\begin{tabular}{|l|l|l|l|l|}
\hline \multirow{2}{*}{} & \multicolumn{2}{|l|}{ Juvenile mortality } & \multicolumn{2}{l|}{ Adult mortality } \\
\cline { 2 - 5 } & full model & separate & full model & separate \\
\hline cold days & $-0.23 \pm 0.19$ & $-0.41 \pm 0.18^{*}$ & $-0.12 \pm 0.24$ & $-0.41 \pm 0.25^{\dagger}$ \\
\hline $\begin{array}{l}\text { population } \\
\text { size }\end{array}$ & $0.19 \pm 0.17$ & $0.35 \pm 0.19^{\dagger}$ & $0.13 \pm 0.22$ & $0.30 \pm 0.27$ \\
\hline $\begin{array}{l}\text { predator } \\
\text { index }\end{array}$ & $0.21 \pm 0.18$ & $0.34 \pm 0.18^{\dagger}$ & $0.56 \pm 0.24^{*}$ & $0.65 \pm 0.23^{* *}$ \\
\hline
\end{tabular}




\section{$590 \quad$ Figure legends}


592 Figure 1. Fitted survival (top) and mortality (bottom) from the preferred model

593 (Logistic, table 1) from day 12 (age 0) up to age 6, after which most individuals in

594 the wild population have died. The grey shaded area indicates the $95 \%$

595 confidence interval around the estimate (solid line).

596 


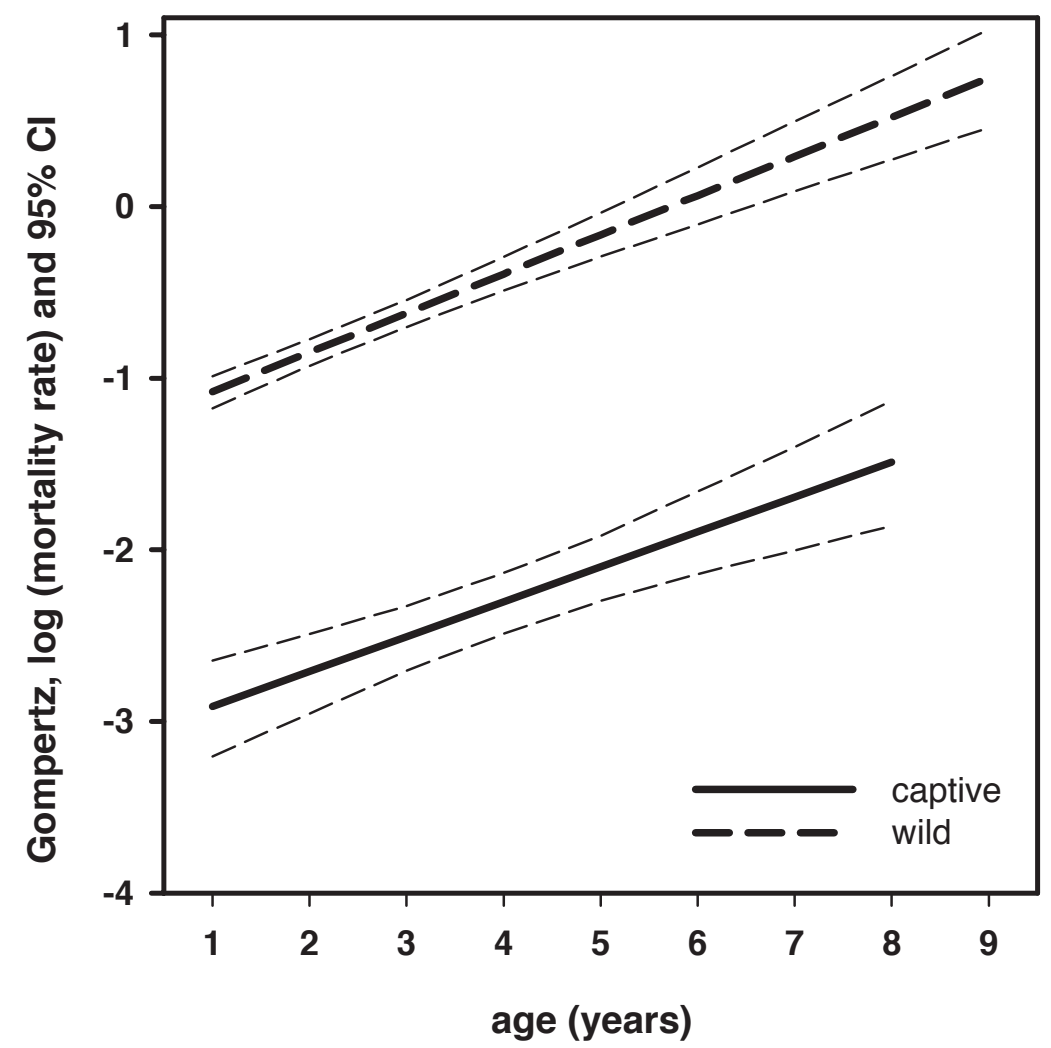

598 Figure 2. Fitted mortality rates under a Gompertz model of house sparrows in

599 the wild and in captivity. Model predictions are plotted across the full range of 600 ages available in the datasets. 


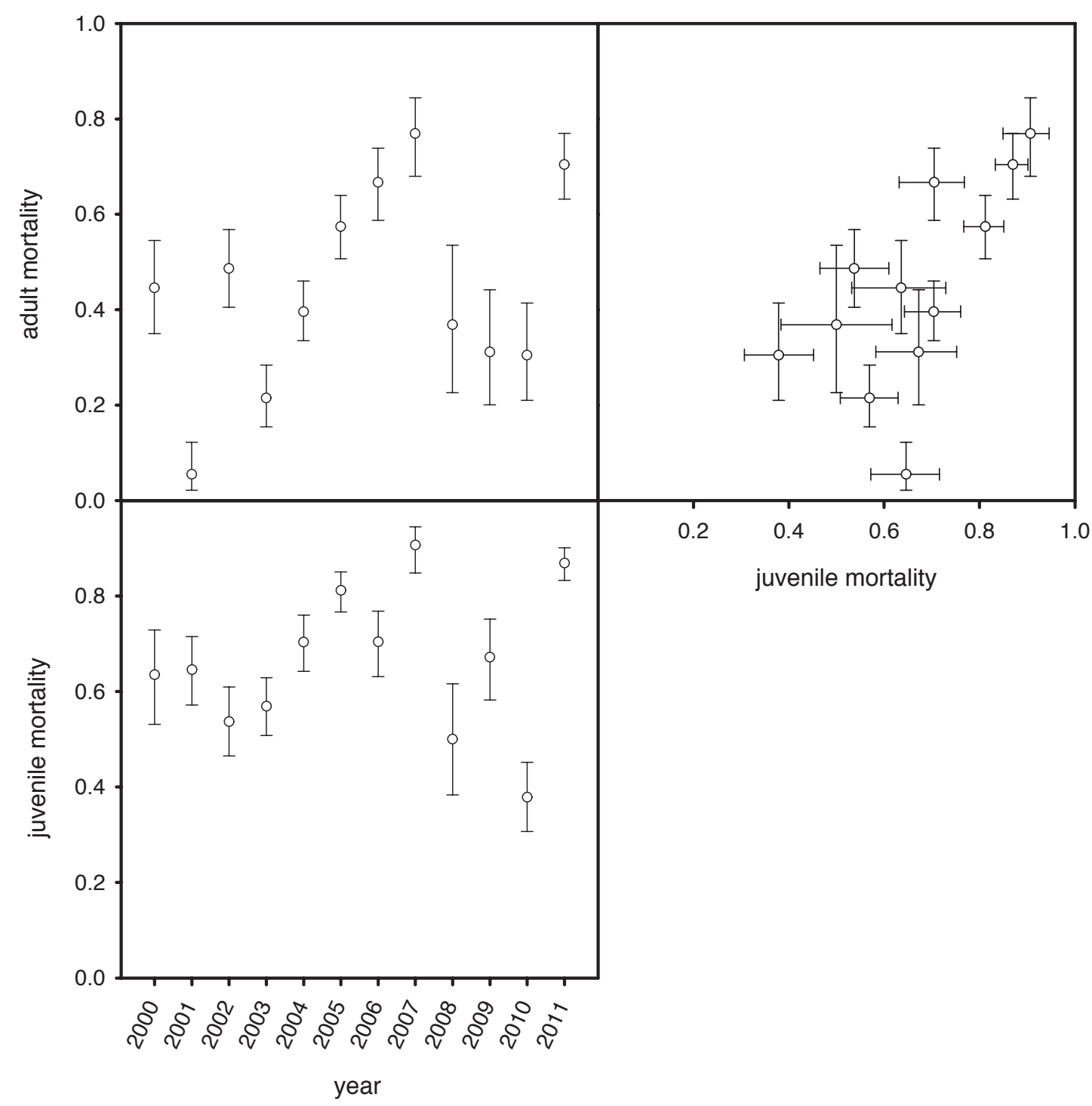

602 Figure 3. Variation in adult and juvenile mortality rates per census year (two

603 graphs on left) and the correlation between these (top right graph). Whiskers

604 indicate confidence intervals of proportions according to Blaker ${ }^{57}$, calculated

605 using the package 'PropCIs' in R. 

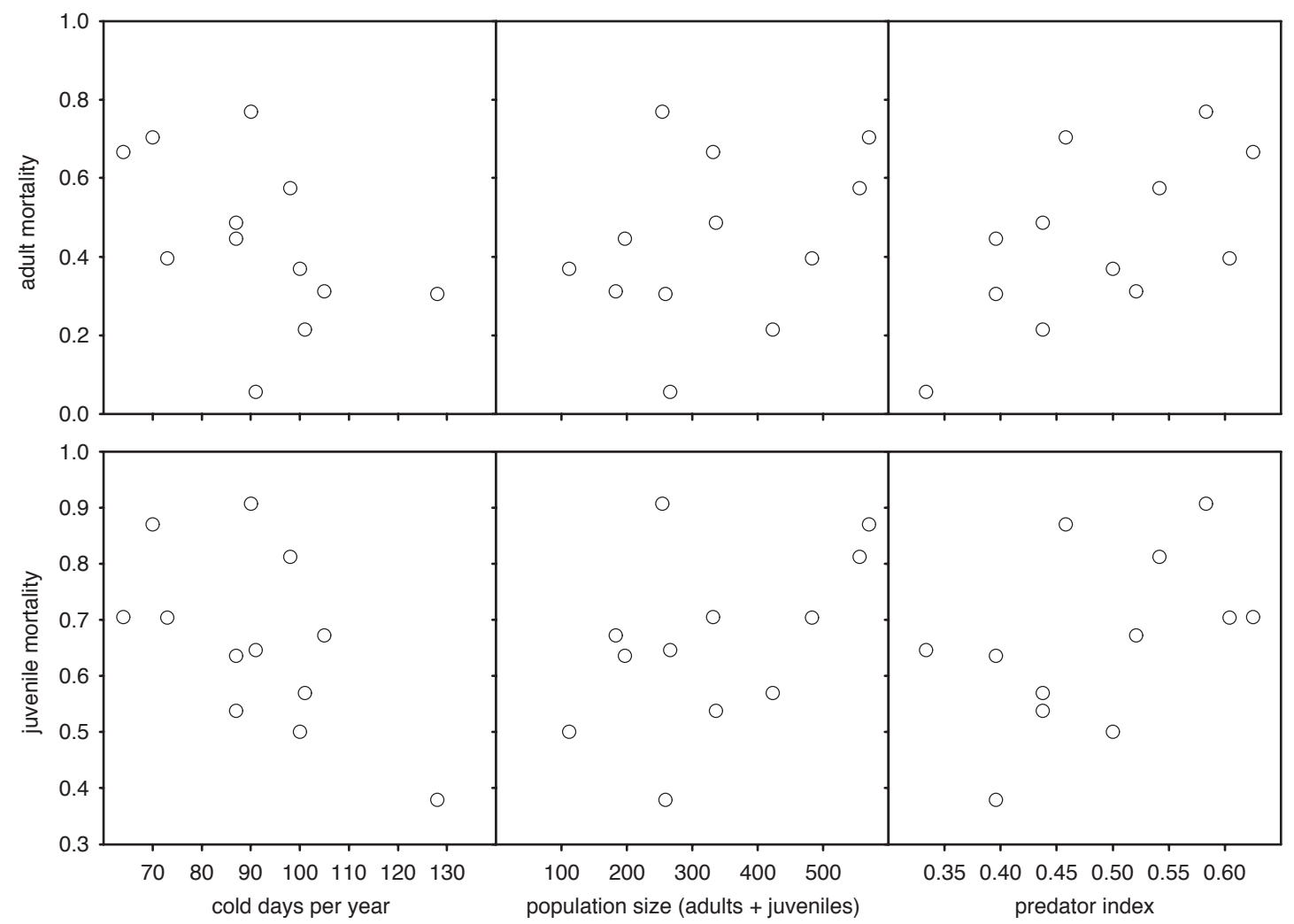

607 Figure 4. Scatter plots between adult or juvenile mortality and temperature,

608 population size or the presence of raptors. Significant relationships were present

609 between predation pressure and both juvenile and adult mortality, and mortality

610 of both juveniles and adults was higher in warmer winters. See text for rank

611 correlations of these relationships and Table 3 for binomial mixed models. 